\title{
Enfrentando as estatísticas: estratégias para permanência de mulheres em STEM
}

\section{Facing the stats: strategies for maintaining women in STEM}

\author{
Letícia Carolina Boffi (orcid.org/0000-0001-9198-8963)1 \\ Ligia Carolina Oliveira-Silva (orcid.org/0000-0002-7487-9420)²
}

\begin{abstract}
Resumo
As mulheres que seguem carreira nas áreas de STEM - Ciencia, Tecnologia, Engenharia e Matemática - vêm sendo estatisticamente sub-representadas no mercado de trabalho e na academia. Fenômenos que ajudam a explicar o cenário são os chamados "efeito tesoura", "teto de vidro" e "leaky pipeline", abordando como as mulheres enfrentam obstáculos que dificultam a permanência em STEM, assim como ascensão a posições de poder. Diante dessas questões, o presente artigo constitui-se num estudo teórico que mapeia e discute estratégias que podem contribuir para o aumento da permanência e ascensão das mulheres em STEM. Conclui-se que tais estratégias englobam ações iniciais tanto na infância e adolescência, no âmbito escolar, quanto na fase adulta, referentes ao ensino superior, aos ambientes de trabalho e à construção do autoconceito da mulher.
\end{abstract}

Palavras-chave: Mulheres. STEM. Carreira. Trabalho. Estratégias.

\begin{abstract}
Women that pursue careers in STEM have been statistically belittled in both the labor market and academia. Phenomena that help explain such a scenario are so-called "scissor effect," "glass ceiling," and "leaky pipeline," which address how women face obstacles that hamper their permanence in STEM, as well as their rise to power positions. Considering these issues, this article is a theoretical study that maps and discusses strategies that may contribute to increase the permanence and rise of women in STEM. We conclude that such strategies include initial actions within schools in childhood and adolescence, as well as in adulthood, concerning higher education, work environments and the construction of women's self-concept.
\end{abstract}

Keywords: Women. STEM. Career. Work. Strategies.

\footnotetext{
${ }^{1}$ Universidade de São Paulo, Ribeirão Preto, Brasil. E-mail: leticiaboffi@gmail.com.

${ }^{2}$ Universidade Federal de Uberlândia, Uberlândia, Brasil. E-mail: ligiacarol@ufu.br.
} 
A presença das mulheres nas carreiras de STEM (Ciência, Tecnologia, Engenharia e Matemática) tem sido alvo constante de pesquisas nos últimos anos. Isso se deve a um aglomerado de fatores, dentre os quais estão os movimentos feministas e a maior abertura das universidades e do mercado para as mulheres (Klanovicz, 2016). Contudo, tal participação ainda é pequena quando comparada à dos homens, de forma que as mulheres se veem sub-representadas nessas áreas (Klanovicz, 2011). As questões que envolvem mulheres e carreiras ditas predominantemente masculinas não se baseiam somente no âmbito educacional, mas envolvem e refletem contextos sociais e culturais de gênero e segregação, acarretando desigualdades (Alves, 2017). Historicamente, desde a sua colocação no mercado de trabalho, a mulher precisou enfrentar diversas lutas para conquistar seus objetivos e se desvencilhar da ideia arcaica de que sua função era apenas ser reprodutora, cuidadora da prole e do lar, o que a mantinha confinada ao ambiente privado (Paiva, 2017).

Os estudos de gênero se fortalecem com a segunda onda do feminismo, em 1970, que traz à tona a luta contra as desigualdades sociais e culturais. Há um aumento de debates sobre como a cultura machista/sexista, as definições de papéis sociais sobre o que é ser homem e mulher e as funções desempenhadas por cada um resultam de uma construção social inserida em um contexto socioeconômico e um período histórico (Klanovicz, 2016). A escolarização das mulheres deu-se tardiamente, quando comparada à dos homens, além de que eles eram incentivados a estudar e trabalhar para adquirir conhecimentos ditos científicos, enquanto as mulheres estudavam como ser uma boa dona de casa, bordados e afazeres domésticos. Isso propiciou maior segregação, além de atraso na educação feminina, pois o domínio das ciências era entendido como destinado apenas aos homens, considerados objetivos, neutros e capazes o suficiente para exercerem o raciocínio necessário para tal (Alves, 2017).

Embora dados brasileiros recentes (Conselho Nacional de Desenvolvimento Científico e Tecnológico [CNPq], 2016) indiquem que as mulheres apresentam mais escolaridade que os homens - entre 20 e 59 anos, as mulheres mestres são mais de $50 \%$ do total, assim como, a partir de 1998 até 2016, mais da metade dos títulos de mestres são para mulheres -, eles são maioria nas áreas de Ciências, Engenharias, Tecnologia e Matemática (Lombardi, 2003), de forma que as mulheres ainda são sub-representadas nas carreiras de STEM (Clerc \& Kels, 
2013; Herman, 2015). Logo, apesar da maior abertura à educação, nos dias atuais ainda se constata a predominância do direcionamento das mulheres para as carreiras das humanidades, principalmente no magistério - carreira que abre o ensino superior às mulheres, mas que também reafirma a colocação de características compreendidas como femininas, como o cuidado e a aprendizagem (Alves, 2017). Isso resulta em números poucos expressivos na participação das mulheres nas áreas de STEM.

A inserção da mulher nas áreas de Ciência e Tecnologia no Brasil não quer dizer que estas convivam com um ambiente igualitário e sem discriminação. Vongalis-Macrow (2016) explicita algumas causas da não permanência das mulheres nas carreiras predominantemente masculinas, tais quais: sexismo (comentários depreciativos, estereótipos, assédio), lacunas de oportunidades, salários menores, menos ofertas de promoções e menor prestígio na sua carreira. Lombardi (2003) reitera as diferenças no lugar de ocupação dentro até mesmo das engenharias, contexto em que as mulheres se encontram mais presentes na Engenharia Química e menos na Mecânica e na Metalurgia. A sub-representação das mulheres nas áreas de STEM pode ser compreendida de duas formas: A primeira refere-se diretamente à pequena quantidade de mulheres em determinadas áreas, como as engenharias, e a segunda apresenta um sistema intrincado no qual a mulher raramente chega à ocupação de cargos de prestígio, mesmo nas carreiras denominadas femininas (Bilimoria, Joy \& Liang, 2008).

Lima, Braga e Tavares (2015) comparam o número de bolsas de produtividade concedidas pelo CNPq em 2015, constatando que as mulheres estão acima dos 60\% nas áreas de Saúde, Humanas e Linguística, Letras e Artes, embora representem apenas 30\% nas áreas das Ciências Exatas e da Terra e nas Engenharias e Computação. Com relação às bolsas concedidas aos pesquisadores que já se encontram no topo de suas carreiras, nos níveis $1 \mathrm{~A}$ e 1B o percentual de mulheres, em 2013, chega a 39\%, enquanto $61 \%$ dos homens recebem as bolsas nessas modalidades. Ou seja, os dados apontam que a quantidade de bolsas concedidas às mulheres ao longo da carreira de pesquisadoras decai à medida que estas avançam na carreira, ilustrando (a) maior dificuldade impostas às mulheres para que cheguem a esses níveis e (b) menor reconhecimento e invisibilidade do trabalho daquelas que se encontram nos níveis mais altos da carreira. Contudo, o Brasil ainda alcança uma 
marca histórica em que as mulheres constituem $49 \%$ dos pesquisadores do país no período analisado entre 1996-200 e 2011-2015 (Elsevier, 2017). Muzi e Luz (2010) refletem sobre a presença das mulheres na ciência e defendem que esse processo exige mudanças estruturais na cultura, método e conteúdo da ciência. Portanto, inicialmente, a ciência foi construída em moldes que atendiam aos homens e suas rotinas dentro do espaço público, de modo que, estruturalmente, a ciência não está preparada para corroborar com a permanência das mulheres, mas sim para excluí-las. Dessa forma, a maior dificuldade das mulheres em STEM é, ainda, a adequação de seus papéis impostos socialmente dentro da esfera privada - mãe, dona de casa - para com a produção científica exigida.

Diante desse cenário, destaca-se a necessidade de se mapear quais fatores podem contribuir para a permanência e ascensão das mulheres em STEM. Vários fatores já foram identificados como sendo responsáveis por dificultarem a permanência das mulheres nessas carreiras. Santos (2016), por exemplo, investiga uma das principais variáveis que influenciam as mulheres e aumentam as dificuldades para se estabilizar na ciência ou avançar na construção da carreira: a maternidade. Adicionalmente, as atividades domésticas ainda são majoritariamente conferidas às mulheres, incluindo a criação e cuidado com os filhos. Isso reduz o tempo disponível para as pesquisas, dificultando as publicações e o avanço, prestígio e reconhecimento na carreira (Santos, 2016). Outras questões associadas à não-permanência das mulheres em STEM são ausência de incentivo escolar, o assédio sexual no trabalho e a discriminação sexual (Amorin, Dantas, \& Carvalho, 2017; Hill \& Silva, 2005; Santos, 2016; Sandler, 2005). Todavia, ainda são raras as discussões que identificam as variáveis que efetivamente favorecem a permanência das mulheres na graduação e carreiras majoritariamente masculinas.

Considerando tal lacuna, o presente artigo tem como objetivo realizar um estudo teórico sobre os fenômenos que permeiam a escassez das mulheres em STEM e, principalmente, o mapeamento das variáveis que podem favorecer a entrada e permanência das mulheres nas carreiras em STEM. Dessa maneira, busca-se possibilitar a realização de mais estudos que incentivem as mulheres a construírem uma carreira longa e promissora nas áreas de STEM, além de fornecer bases para intervenções que mitiguem a sub-representação delas nestas áreas, contribuindo para o aumento da equidade de gênero. 


\section{Fenômenos que permeiam a escassez de mulheres em STEM}

Alguns dos principais motivos para a escassez de mulheres acima de 35 anos nas áreas de STEM são a ausência ou precariedade de perspectivas de promoção na carreira, além da diferença de salário em razão do gênero (Vongalis-Macrow, 2016). Considerando que carreira representa o padrão de experiências de trabalho ao longo do curso de vida de um indivíduo (Greenhaus, Callanan, \& Godhsalk, 2010), percebe-se que o conceito de carreira vai além dos vínculos empregatícios formais, envolvendo aspectos como estudos, atividades autônomas e trabalho voluntário. Sendo assim, é necessário compreender que a carreira representa algo contínuo, cumulativo e de longo prazo, que acompanha o indivíduo ao longo de toda sua vida laboral. Nesse sentido, no que diz respeito à carreira de mulheres, Lima, Carvalho Neto, Lima, Tanure e Versiani (2013) evidenciaram que para alcançar uma carreira executiva, as mulheres necessitam investir proporcionalmente mais em seus trabalhos, demonstrar maior comprometimento e despender mais esforços que os homens. Outras pesquisas indicam que histórias de sucesso de professoras universitárias de Física são pautadas na experiência de desconstrução de gênero na educação durante a infância e seu percurso na carreira (Amorin et al., 2017). Contudo, isso não significa que tais profissionais não esbarraram na segregação de gênero nas disciplinas, além de preconceitos, discriminações e relações hierárquicas implícitas e explicitas. Em sua pesquisa, Falk, Rottinghaus, Casanova, Borgen e Betz (2017) encontraram que 60\% das mulheres nas áreas de STEM apresentavam baixo nível de interesse e confiança, apesar de já terem entrado nas áreas. Evidenciou-se que baixas expectativas com a faculdade, combinadas a situações nas quais homens explicam matérias às mulheres, representam uma mensagem para as estudantes de que o envolvimento em STEM é uma coisa masculina e que, portanto, a assistência dos homens é necessária para se obter sucesso na área (Falk et al., 2017).

Entre os fenômenos que refletem esse cenário, um dos mais citados na literatura é o "teto de vidro", que corresponde ao progresso das mulheres jovens em suas carreiras até alcançarem uma determinada posição na hierarquia, quando estruturas e processos não explícitos em formas de desvantagens apresentam-se como barreiras intransponíveis ao avanço para 
posições mais elevadas (Altman, Simpson, Baruch, \& Burke, 2005). Esse fenômeno representa a dificuldade de as mulheres ocuparem altos cargos na hierarquia organizacional, incluindo a chefia, apesar de apresentarem educação apropriada e aprimoramento ao longo dos anos de serviço. Morrison e Von Glinow (1990) descrevem tal fenômeno como sendo uma barreira tão sutil que é transparente, mas ainda tão forte que impede que as mulheres e as minorias se movam na hierarquia de gerenciamento. Os processos seletivos representam um dos mecanismos que agregam para o fortalecimento do teto de vidro, uma vez que sua complexidade e subjetividade muitas vezes contribuem para que a discriminação seja encoberta (Lima et al., 2013). A perspectiva sexista implícita, frequentemente, desfavorece a contratação ou promoção de mulheres para cargos de chefia, pois considera que haverá gastos com os quais a organização deverá arcar caso a mulher engravide, por exemplo. Tal raciocínio representa um viés inconsciente, que favorece a continuidade dos preconceitos e da discriminação de gênero (Lima et al., 2013).

Outro fenômeno frequentemente citado na literatura é o "/eaky pipeline" ou cano que pinga, segundo o qual o mercado de trabalho seria como um oleoduto e a representatividade feminina, em STEM, estaria intimamente ligada à quantidade de mulheres que, de fato, adentram mercados dominados pela presença masculina (Schweitzer, $\mathrm{Ng}$, Lyons, \& Kuron, 2011). Pipeline é descrito como o processo de contínua aprendizagem e interesse que envolve as áreas das engenharias, matemática, ciência e tecnologia, promovendo maiores políticas de educação nessas disciplinas (Lyon, Jafri, \& Loius, 2012). Profissionais do gênero feminino bem treinadas e qualificadas estariam evaporando ao invés de ascender para posições bem remuneradas de liderança e autoridade. Ou seja, o leaky pipeline ou "cano que pinga" corresponderia ao fenômeno de diminuição de mulheres à medida que se ascende para posições mais elevadas na carreira.

Algumas evidências reforçam a premissa de que o fenômeno se mostra desde muito cedo e em alguns períodos decisivos, tais quais: na escolha da carreira ao fim do ensino médio; na passagem da graduação à pós-graduação ou desistência; depois da graduação, diante da não aprovação em processos seletivos ou como docente universitária e na ausência de disposição para concorrer a cargos de direção no ambiente de trabalho (Saavedra, Taveira, \& Silva, 2010). Segundo revisão de Blickenstaff (2005), os principais motivos para o 
"vazamento" de mulheres dos campos de STEM seriam: a falta de experiências positivas com a ciência na infância; a ausência de profissionais do sexo feminino como modelos; o favorecimento dos estudantes do sexo masculino na pedagogia das aulas de ciências; a existência de um ambiente hostil para meninas/mulheres nas aulas de ciências; a pressão cultural sobre meninas/mulheres para se adequarem aos papéis tradicionais de gênero e a visão predominantemente masculina da epistemologia científica.

Um terceiro fenômeno que envolve a presença de mulheres em STEM é o efeito tesoura. De maneira similar ao leaky pipeline, o efeito tesoura "corta" as mulheres para fora de suas carreiras à medida que estas avançam na mesma, ou seja, à medida que as mulheres avançam, constata-se uma gradativa perda de espaço que não advém necessariamente da meritocracia (Brito, Pavani, \& Lima Jr., 2015). O efeito tesoura considera a inversão da predominância de um grupo sobre outro, isto é: as mulheres seriam maioria durante a etapa de formação, o que se modifica à medida em que se observa as posições de destaque ao longo da carreira. Observa-se esse efeito no Brasil na carreira de Física, em que, dentre todas as ciências, o aumento do número de mulheres tem sido o mais lento (Agrello \& Garg, 2009). Na Física, $30 \%$ dos ingressantes da graduação são mulheres, $20 \%$ seguem para o mestrado e doutorado e apenas 15\% chegam à docência (Brito et al., 2015). Tal fenômeno também está presente na concessão de bolsas de produtividade (PQ), ao passo que, do total de bolsas concedidas pelo CNPq em 2015, apenas 36\% foram concedidas a mulheres (Lima et al., 2015).

\section{Fatores que favorecem a permanência de mulheres em STEM}

Diante do cenário descrito, buscou-se identificar ações que influenciassem, principalmente, a permanência das mulheres das áreas de STEM. Ações que resultam no abandono das carreiras devem ser levadas em consideração como pontos a serem evitados, entretanto, aquelas que preveem o aumento da entrada de mulheres nessas áreas e influenciam a permanência e ascensão na carreira foram priorizadas. Tais ações foram subdivididas de acordo com as fases do desenvolvimento humano, de forma que abordaremos primeiro as 
iniciativas na infância e adolescência, para, posteriormente, apresentarmos as que podem ser efetuadas já na fase adulta.

\section{Iniciativas na infância e adolescência}

A ausência ou pouco êxito de estratégias ao longo da infância e adolescência pode levar meninas à desistência e/ou falta de interesse nas áreas de STEM antes mesmo de optarem por um curso técnico ou universitário, o que contribuiria para acentuar a inequidade de gênero. Pesquisas observam que o incentivo à entrada de garotas nas áreas de STEM deve apresentar-se mais cedo do que a faculdade em si (Astin \& Sax, 1996; Huang \& Brainard, 2001; Kinzie, 2007). Velho e Leon (1998) afirmam que até os $12 / 13$ anos de idade, alunos e alunas apresentam desempenho semelhante nas diferentes disciplinas, contudo, depois desse período, são instituídos obstáculos sutis e diretos, sobretudo em relação às meninas que se aproximam das ciências exatas e tecnologias. No ensino fundamental e médio, evidencia-se uma variedade de diferenças entre o ensino de garotos e garotas, o que acaba por afastar as meninas da escolha das carreiras em matemática, ciências, engenharia e tecnologia. A preparação nos estudos de matemática e ciências no ensino fundamental é frequentemente citada como uma importante influência na tomada de decisão na entrada ou saída de mulheres nas áreas de STEM frente à universidade (Shapiro \& Sax, 2011). Essas diferenças continuariam no ensino médio, o que afeta a permanência de mulheres na computação, por exemplo, uma vez que os homens demonstrariam mais conhecimentos avançados quando comparados às mulheres, gerando nestas últimas uma baixa confiança acerca de suas habilidades (Margolis, Fisher, \& Miller, 2000).

As mulheres que não recebem a base matemática e científica necessária para acessar as carreiras de STEM encontram dificuldades consideráveis para continuarem seus estudos nessas áreas (Huang \& Brainard, 2001). Portanto, as diferentes experiências destinadas às meninas e meninos nos anos iniciais da educação constituem uma forte barreira para a permanência de mulheres nas carreiras predominantemente masculinas. Torna-se necessária a observação das diferenças de ensino, além da busca por uma educação que permita que as crianças tenham as mesmas chances e incentivos para seguirem carreiras em 
STEM. A experiência escolar é decisiva na escolha e no incentivo das carreiras das meninas, pois pode tanto reproduzir os valores e estereótipos que separam as garotas das áreas de STEM, quanto contribuir de forma definitiva para a mudança do pensamento delas em relação a si mesmas e suas capacidades (Olinto, 2011). Em suma, a educação deve ser a base da reforma que é necessária quando se objetiva a alteração de padrões vigentes que disseminam a inferioridade feminina e a sua incapacidade na realização de certas atividades. Seguindo essa linha, uma das primeiras influências do feminismo na educação foi a reivindicação do acesso das mulheres à ciência (Rosa \& Silva, 2015). As representações de masculinidade ou feminilidade ligadas aos cursos e profissões afastam ou atraem as meninas ou meninos, afetando sua autoconfiança e autoeficácia (Olinto, 2011). De acordo com Rosa e Silva (2015), a primeira onda do feminismo chama atenção para a ausência de modelos femininos na ciência e, a partir dessa constatação, iniciam-se programas extraclasses para que as garotas adquiram habilidades nesse campo. Logo, a ausência de modelos para garotas dentro das áreas cientificas é vista com uma grande fonte de influência no interesse das mesmas em seguir carreiras em STEM.

Pesquisadoras ressaltam a importância da comunicação promovida pelas mídias, de maneira que esse pensamento pode ser redirecionado à educação (Rosa \& Silva, 2015). Nas séries iniciais, as imagens ocupam grande espaço dos livros didáticos e exercem considerável influência nos alunos ao longo dos anos, sendo necessária mais atenção às figuras circundantes nos materiais utilizados na educação de meninos e meninas. Uma análise dos livros de física, realizada por Rosa e Silva (2015), demonstrou que em todas as categorias analisadas (quadrinhos, atividade física/esporte, profissão, história da ciência e atividade de caráter científico) há maior representação masculina. Nessas representações, os meninos aparecem em situações ao ar livre, enquanto as meninas realizam atividades domésticas. As autoras concluem que os livros didáticos de física analisados reforçam os estereótipos de que as mulheres devem cuidar dos filhos, da casa e de sua aparência física. Dessa forma, observa-se que as mídias que cerceiam as atividades das crianças não apresentam (ou apresentam pouco) modelos femininos em atividades de ciência e tecnologia, o que contribui para reforçar os estereótipos e a segregação das mulheres nas áreas de STEM. Portanto, é necessária atenção à presença de modelos femininos em todas as esferas sociais, 
principalmente àquelas relacionadas às atividades predominantemente masculinas, para que assim as meninas percebam e internalizem a existência de mulheres nessas áreas, e cogitem a possibilidade de ser uma delas.

A necessidade de projetos que incentivem a maior participação de meninas em campos STEM representa outra iniciativa necessária à permanência feminina nessas áreas. Damasceno e Bentes (2014), por exemplo, registraram os resultados da aplicação de um curso extraclasse de tecnologia, engenharia e computação voltado às alunas do ensino médio, objetivando atraí-las às carreiras que envolvam essas áreas. O projeto resultou nas alunas maior conhecimento de informações e, principalmente, aumento do interesse sobre as possibilidades de atuação profissional nas carreiras tecnológicas. Outra inciativa bem sucedida foi a de Brito et al. (2015), que realizaram o projeto "Meninas na Ciência", em 2014, visando a integração universidade-escola e, principalmente, a formação de massa crítica acerca das relações entre ciência e gênero. Alguns dos principais resultados foram a formação continuada dos professores visando aspectos sobre Astronomia e gênero, a realização de oficinas de discussão sobre ciência e gênero e a produção de vários curtas, numa série intitulada "Lugar de Mulher", na qual são apresentadas figuras de referência para meninas e mulheres em STEM. Logo, destaca-se a importância de projetos extracurriculares nas escolas, em parcerias com universidades, que abarquem o ensino das ciências e tecnologias às meninas e que realizem discussões de gênero. Dessa forma, possibilita-se maior conhecimento sobre os assuntos citados e se desperta maior interesse nas garotas para as carreiras em STEM, contribuindo para diminuir a desigualdade de gênero nessas áreas. O Quadro 1 apresenta algumas das iniciativas que foram realizadas no Brasil, por meio de entre órgãos de fomento e ONG's, nos últimos anos. 


\section{Quadro 1}

Programas e Editais para Meninas em STEM nos últimos anos

\begin{tabular}{|c|c|c|c|c|}
\hline Programa & Objetivo & Datas & $\begin{array}{l}\text { Orgãos de } \\
\text { Fomento }\end{array}$ & Ações \\
\hline $\begin{array}{l}\text { Meninas e Jovens } \\
\text { Fazendo Ciências } \\
\text { Exatas Engenharias } \\
\text { e Computação? }\end{array}$ & $\begin{array}{l}\text { Selecionar projetos para apoio } \\
\text { financeiro que visem estimular a } \\
\text { formação de mulheres para as } \\
\text { carreiras de ciências exatas, } \\
\text { engenharias e computação no } \\
\text { Brasil, como também despertar o } \\
\text { interesse de estudantes do sexo } \\
\text { feminino do Ensino Médio e da } \\
\text { Gra duação por estas pro fissões e } \\
\text { para a pesquisa cientifica e } \\
\text { tecnológica. }\end{array}$ & 2013 & $\begin{array}{l}\text { MCTI; } \\
\text { CNPq; } \\
\text { SPM; } \\
\text { Petrobrás }\end{array}$ & $\begin{array}{l}\text { Chamada para apoio } \\
\text { financeiro a projetos que } \\
\text { pesquisa que contribua } \\
\text { para os objetivos do } \\
\text { programa. }\end{array}$ \\
\hline $\begin{array}{l}\text { Meninas e Jovens } \\
\text { Fazendo Ciência, } \\
\text { Tecnologia e } \\
\text { Inovação }{ }^{2}\end{array}$ & $\begin{array}{l}\text { Edital cujo objetivo é selecionar } \\
\text { pesquisas para apoio financeiro } \\
\text { que contribuampara o } \\
\text { desenvolvimento científico e } \\
\text { tecnológico e a inovação do } \\
\text { País. Visa também ampliar o } \\
\text { número de estudantes do sexo } \\
\text { feminino nas carreiras de } \\
\text { ciências exatas, engenharias e } \\
\text { computação. }\end{array}$ & 2014 & $\begin{array}{l}\text { MCTI; } \\
\text { CNPq; } \\
\text { Petrobrás }\end{array}$ & $\begin{array}{l}\text { Com investimento de RS } \\
10,9 \text { milhões (concessões } \\
\text { de bolsas e custos } \\
\text { operacionais) selecionou } \\
325 \text { projetos do total } \\
\text { de } 528 \text { apresentados para } \\
\text { apoiar financeiramente. }\end{array}$ \\
\hline $\begin{array}{l}\text { Meninas nas } \\
\text { ciências Exatas, } \\
\text { Engenhariase } \\
\text { Computação? }\end{array}$ & $\begin{array}{l}\text { O objetivo da chamada é "apoiar } \\
\text { projetos que visem a formação } \\
\text { de mulheres para as carreiras de } \\
\text { ciências exatas, engenharias e } \\
\text { computaçãono Brasil". }\end{array}$ & 2018 & $\begin{array}{l}\text { CNPq; } \\
\text { MICTI }\end{array}$ & $\begin{array}{l}\text { Financiamento de projetos } \\
\text { de pesquisas em escolas. }\end{array}$ \\
\hline Elasnas Exatas ${ }^{4}$ & $\begin{array}{l}\text { O programa apoia iniciativas que } \\
\text { favoreçama inserção das } \\
\text { meninas nas áreas de ciências } \\
\text { tecnológicas e exatas por meio } \\
\text { da promoção de equidade de } \\
\text { gênero. }\end{array}$ & 2015 & $\begin{array}{l}\text { Fundo } \\
\text { ELAS; } \\
\text { Instituto } \\
\text { Unibanco; } \\
\text { Fundação } \\
\text { Carlos } \\
\text { Chagas; } \\
\text { ONU } \\
\text { Mulheres }\end{array}$ & $\begin{array}{l}\text { Edital para seleção de } \\
\text { projetos que estimulemas } \\
\text { meninas a se envolverem } \\
\text { com as ciências exatas e } \\
\text { tecnológicas. }\end{array}$ \\
\hline
\end{tabular}

Outro fator de grande influência para o aumento e permanência feminina em STEM é a família. Modelos familiares (incluindo-se pais e mães) costumam ser fatores altamente responsáveis por influenciar o desejo das meninas para entrar em STEM durante o colégio (Shapiro \& Sax, 2011), de forma que é mais provável que meninas sigam as carreiras de STEM se ambos os pais já estiverem nestas profissões (Astin \& Sax, 1996). É importante, portanto, a presença de modelos familiares que possam ser referenciais para as meninas no colégio, incluindo mulheres em sua própria família. Olinto (2011) ainda sugere a necessidade da redução dos estereótipos de gênero dentro de casa, visando à diminuição da influência destes nas escolhas profissionais das meninas. Evidências apontam que, ao longo dos anos escolares, as meninas costumam ser mais propensas a perceber a carreira em STEM como incompatível com a formação "bem sucedida" de uma família, sendo tal fator 
mais uma barreira para a escolha da área (Hawks \& Spade, 1998). Logo, torna-se necessária a discussão com as garotas acerca das concepções de família "bem sucedida", além da desconstrução da fantasia de que uma família requer dedicação exclusiva, para que assim seja possível que elas ingressem nas carreiras que desejam, sem perceber a constituição de uma família como empecilho. Infelizmente, iniciativas na infância e adolescência podem não ser suficientes para diminuir a predominância masculina em áreas de STEM. Sendo assim, iniciativas na fase adulta também são necessárias, principalmente porque a entrada na universidade e, posteriormente, no mercado de trabalho, costuma acentuar a percepção de barreiras.

\section{Iniciativas na fase adulta}

Mulheres que optam por uma carreira em STEM, evidentemente, superaram obstáculos ao longo de sua infância e adolescência, logo, o ambiente acadêmico e/ou profissional deveria ser acolhedor para aquelas que conseguem transpor as barreiras sociais e os preconceitos acerca da decisão de carreira. Entretanto, poucas encontram apoio e sororidade nesses contextos que, frequentemente, apresentam uma nova gama de barreiras a serem enfrentadas. Segundo Olinto (2011), dois mecanismos de barreiras podem ser identificados na segregação das mulheres: a segregação horizontal e a vertical. Na segregação horizontal, aparecem mecanismos que direcionam as escolhas das carreiras das mulheres em função do gênero, ou seja, as mulheres são levadas a realizarem escolhas profissionais de acordo com sua autoavaliação, o que pode apontar para maior preferência por carreiras e atividades as quais elas acreditam ou foram levadas a acreditar que são mais adequadas. Na segregação vertical, ocorrem processos invisíveis que não permitem a ascensão das mulheres em suas carreiras (teto de vidro, leaky pipeline e efeito tesoura), mantendo-as em posições hierárquicas subordinadas ou intermediárias. Diante dessas situações, muitas barreiras não são explícitas, porém, detectáveis. Outro fator que merece atenção é a ameaça do estereótipo, que ocorre quando alguém que pertencente a um grupo estereotipado faz algo que confirma o estereótipo negativo dado a esse grupo. Portanto, as mulheres estariam 
internalizando os estereótipos socialmente dirigidos a elas, o que também afetaria a entrada e permanência nas carreiras em STEM.

Nesse sentido, é preciso distinguir as estratégias internas das externas, refinando-se as possibilidades de combate aos fenômenos que são ainda mais evidentes nas áreas de STEM. Em relação às estratégias internas, estas se direcionam aos fatores psicológicos que afetam a percepção das mulheres sobre seu autovalor e capacidades. Uma das razões pelas quais há diferenças entre homens e mulheres na participação em STEM é que as mulheres tendem a subestimar suas habilidades que a levarão ao sucesso nessas carreiras (Ehrlinger \& Dunning, 2003). Evidências apontam que, frequentemente, as mulheres se desvinculam das suas carreiras na ciência e na engenharia não por falta de conhecimento, mas sim por apresentarem pouco senso de autoconfiança (Brainard \& Carlin, 1998), havendo uma correlação positiva entre a percepção das barreiras por mulheres e o baixo nível de autoeficácia e interesse por STEM (Cadaret, Hartungb, Subich, \& Weigold, 2017). Falk et al. (2017) demonstram que as mulheres não só apresentam baixo nível de autoeficácia, como também reportam poucas estratégias de enfrentamento. O desenvolvimento do senso de autoeficácia representa uma relevante estratégia interna, possibilitando o enfrentamento das situações adversas. Pessoas com maior autoeficácia acreditam que são capazes de mobilizar sua motivação, seus recursos cognitivos e cursos de ação necessários para realizarem com sucesso suas tarefas, optando por iniciativas mais desafiantes e sendo perseverantes diante de barreiras (Luyhans, Yousseff, \& Avolio, 2007).

Com relação às estratégias externas, estas dizem respeito a fatores passíveis de intervenção exterior. A cultura por trás dos cursos de ciências tem sido identificada como um importante fator de influência na seleção e sucesso nas áreas de STEM (Shapiro \& Sax, 2011). É comum que as mulheres experienciem um clima frio e hostil ao longo da sua vida acadêmica (Amorin et al., 2017), somado ao fato de que a natureza competitiva dos cursos é um aspecto pedagógico que pode manter ou afastar as mulheres (Seymour \& Hewiti, 1997). As interações dentro do ambiente acadêmico também devem ser levadas em consideração, pois as mulheres tendem a não se identificar com a natureza masculina das ciências e da matemática, devido às mensagens implícitas e explícitas, que incluem estereótipos que reforçam a incapacidade das mulheres de realização de certas tarefas (Nosek, Banaji, \& 
Greenwald, 2002). Em suma, a diferenciação de gênero vivenciada pelas meninas durante a formação em STEM resulta em estereótipos negativos, baixas expectativas depositadas nelas e até mesmo assédio ao longo da trajetória acadêmica (Sandler, 2005). Considerando esse cenário, Sandler (2005) propôs um guia para educar gestores e professores, visando identificar e suprimir comportamentos cotidianos que exponham de alguma forma as mulheres, tais como: exclui-las da participação em reuniões e conversas, tratá-las de forma diferenciada quando seus resultados são iguais, dedicá-las menos atenção e estímulo intelectual, desmotivá-las a proclamar o poder masculino. Ainda com relação a aspectos culturais, Busch-Vishniac (2015) destaca que compreender a cultura da organização e seus valores é essencial para que mulheres se mantenham na carreira em STEM. Reconhecer o que mais importa numa empresa é fundamental para ser reconhecida e avançar para os patamares mais elevados, assim como estabelecer uma boa reputação e tomar os devidos créditos pelas próprias ideias.

Outra estratégia externa que também considera o contexto é o estímulo à liderança, cujas oportunidades podem existir em todos os níveis das organizações (McCauley \& Lee, 2015). Entretanto, é comum que as mulheres apresentem dificuldades para desenvolverem seu potencial de liderança, uma vez que com frequência não acreditam que podem, apresentam percepção limitada de oportunidades ou falha no reconhecimento delas e não reconhecem sua própria força (McCauley \& Lee, 2015). Logo, o desenvolvimento da liderança é essencial para alteração da visão das mulheres sobre elas mesmas e o contexto na qual estão inseridas. A liderança apresenta características que permitem seu treinamento e desenvolvimento, tais como: consultar outras pessoas antes de fazer planos ou tomar decisões; desenvolver uma rede de relacionamentos profissionais; delegar responsabilidades; desenhar objetivos, estratégias e procedimentos para o alce das metas; afetar pessoas em posições hierárquicas mais altas; identificar, analisar e atuar para a resolução de problemas; motivar outras pessoas; promover reconhecimento quando apropriado; exercitar as habilidades de outras pessoas (Catalyst, 2007). Portanto, em qualquer posição hierárquica, seria possível apresentar comportamentos de liderança que resultam em benefícios nas relações, o que poderia, consequentemente, contribuir para o avanço da carreira de mulheres em STEM. 
Outra estratégia relativa ao local de trabalho diz respeito à presença de suporte. Uma pesquisa de Fouad, Singh, Cappaert, Chang e Wan (2016) demonstrou que a ausência ou presença de suporte no local de trabalho é um fator que influencia a permanência das mulheres em organizações, pelo menos na área da engenharia. Os resultados mostram que as mulheres que permanecem em seus empregos apresentam como argumento o suporte oferecido no local de trabalho, traduzido em melhor provisão de avanço na carreira e maior compreensão de seus chefes quanto à possibilidade de balancear seu trabalho e seu papel familiar. Dessa forma, constata-se que os locais de trabalho, as possibilidades de crescimento e a forma que essas mulheres são percebidas e consideradas nos mesmos são grandes fatores de influência na sua decisão de continuar ou abandonar a carreira. Assim como na infância e adolescência, a presença de modelos representa uma importante estratégia para a permanência de mulheres adultas em STEM. Para Astin e Sax (1996), estudantes que se veem diante de modelos dentro das ciências apresentam maior probabilidade para seguir suas aspirações científicas, contudo, nota-se que as mulheres têm acesso limitado a modelos e mentores femininos. Diante disso, é imperativa a apresentação de mulheres importantes e/ou que participaram da construção das áreas de STEM, para que as jovens que se encontram na graduação possam sentir-se motivadas e encorajadas a permanecer no curso. Cheryan, Siy, Vichayapa, Drury e Kim (2011) evidenciam que o contato, mesmo que breve, com um membro do mesmo gênero no campo de trabalho pode moldar as crenças de estudantes acerca de suas próprias habilidades e potencial de sucesso no campo em questão. Estudos revelam que os modelos que apresentam estereótipos próprios do campo afetam as crenças das mulheres acerca de seu sucesso; infelizmente, os estereótipos propagados pelas mídias nas áreas de STEM não correspondem à realidade das mulheres que alcançam o sucesso. Na física, por exemplo, Barthlemey, Mccormick e Henderson (2016) apontam que muitas mulheres tendem a se masculinizar para serem mais aceitas pelos seus pares na busca de confiabilidade. Tais questões salientam a importância da apresentação de modelos femininos nas áreas de STEM, para que as mulheres se enxerguem nessas carreiras e não necessariamente entendam que devem internalizar comportamentos masculinos para serem aceitas e respeitadas. 
A construção de uma rede de relacionamentos representa mais uma estratégia que contribui para a permanência de mulheres em STEM. Para Rummer (2015), a rede de relacionamentos pode ser entendida como o ato de se ligar a outras pessoas e seus campos de atuação, sendo tais conexões com pessoas que apresentem os mesmos interesses que o indivíduo compartilha e se mantendo necessariamente uma comunicação. Essa rede facilita a transferência de conhecimentos, tecnologias e habilidades, o que leva a mulher a relacionarse mais com profissionais que são benéficos para sua carreira. A rede de relacionamentos também serve para apoiar as profissionais em momentos difíceis e ajudar na instrumentalização para o avanço da carreira, podendo conter pessoas mais próximas, mas também colegas distantes, colaboradores e supervisores (Rummer, 2015). Outros fatores importantes são a amplitude e a eficácia das pessoas que compõem a rede, porquanto, essas facilitarão ou não o alcance de objetivos por meio, por exemplo, do recebimento de informações mais especificas necessárias para o trabalho (Rummer, 2015). Outras pesquisas afirmam que as redes de relacionamentos pessoais de homens e mulheres apresentam função e composição distintas (Reuben, Rey-Biel, Sapienza, \& Zingales, 2012; Vanbrabrant, 2012). As redes de relacionamentos das mulheres contêm mais familiares, são mais diversas, mais íntimas e menores em quantidade, além de que as pessoas tendem a ser mais apoiadoras, colaboradoras e mais aptas a utilizarem as mídias sociais. Por fim, as mulheres devem-se atentar aos eventos de desenvolvimento de redes, seminários e workshops, tomando vantagem das lições ensinadas, assim também como das pessoas apresentadas (Rummer, 2015).

Outra maneira de aumentar a permanência das mulheres nas áreas de STEM seria a mentoria, definida como a relação positiva entre um sujeito confiável e que tenha experiência e um indivíduo menos experiente ou um grupo de pessoas que facilitem o desenvolvimento profissional e, às vezes, pessoal de uma pessoa inexperiente (Grant, 2015). Isso empodera o sujeito inexperiente a seguir em frente, atribuindo-Ihe confiança ao longo da construção de sua carreira por meio de suporte, encorajamento, conselhos e fornecimento de informações (Grant, 2015). Nas carreiras de STEM, torna-se essencial conhecer as novidades tecnológicas e as questões globais que as cercam; sendo assim, a mentoria representaria uma oportunidade de as profissionais terem acesso às informações 
atuais, detalhes sobre tecnologia de ponta e áreas emergentes em pesquisa, pontos fundamentais para aumentar a eficácia de decisões sobre suas carreiras. A mentoria representa uma estratégia que pode promover o sucesso de uma profissional em STEM, uma vez que facilita a compreensão das dinâmicas políticas, sociais e econômicas associadas a essas áreas (Grant, 2015). Brainard e Carlin (1998) apontam resultados positivos em programas formais que designam uma mentora para uma mulher aprendiz. Ao conectar uma mulher mais experiente a uma mais nova na área da engenharia, notou-se o aumento da permanência das mulheres no curso. Da mesma maneira, ajudar outras mulheres, sendo mentoras delas, produz recompensas importantes (Busch-Vishniac, 2015).

Paralelamente à mentoria, o aconselhamento ou orientação de carreira representa uma estratégia de destaque para a permanência de mulheres em STEM. Para Pritchard (2015), o sucesso de mulheres em STEM envolve coragem e disposição para a realização de escolhas difíceis, de maneira que o aconselhamento de carreira deve abarcar tanto essas questões quanto o planejamento contínuo da carreira. Muitas mulheres nas áreas de STEM não planejam suas carreiras, nem sempre pensam nos passos a serem dados ou aplicam conhecimentos formais de treinamentos. O processo de orientação de carreira deve contribuir para o desenvolvimento da gestão da própria carreira, estimulando o autoconhecimento, a identificação de oportunidades, as decisões planejadas e a implementação e revisão periódica do projeto de carreira (Pritchard, 2015). O aconselhamento de carreira utilizando uma abordagem feminista contribui para o empoderamento das mulheres, principalmente porque considera o contexto em que elas vivem (Chronister, McWrither, \& Forrest, 2008). Destaca-se, portanto, a necessidade de se observar o contexto da mulher, definindo, primeiramente, seu conceito de sucesso e, posteriormente, discutindo os valores sociais vigentes, pois dessa forma é possível compreender melhor suas metas. Para Busch-Vishniac (2015), o avanço de mulheres nas carreiras em STEM implica em decidir adequadamente sobre uma oportunidade de emprego e deliberar sobre a continuidade da carreira. A orientação de carreira contribuiria, dessa forma, para o delineamento de metas especificas, assim como para a flexibilidade, permitindo revisá-las de acordo com a maturidade da profissional e as mudanças de objetivos. 
As estratégias podem ser aplicadas no contexto de programas e editais de agências de fomento específicos para mulheres, cujo objetivo é incentivar a entrada e permanência destas em STEM e oferecer mais visibilidade para as mulheres cientistas, o que representa um movimento da contemporaneidade (Caseira \& Magalhães, 2015). Esses programas são importantes, pois, em sua maioria, buscam também a equiparação de homens e mulheres nas ciências, promovem debates em relação à igualdade de gênero e uma ampla divulgação do conhecimento científico (Caseira \& Magalhães, 2017). Isso torna a questão um problema reconhecido como de ordem pública e social e que, portanto, suas ações fazem parte da busca de solução do mesmo. Conforme disposto no Quadro 2, o programa "Mulher e Ciência" é um exemplo importante nas politicas públicas que envolvem equidade de gênero, sendo considerado um marco por avançar nas discussões de gênero dentro da ciência, tecnologia e estudos feministas (Lima \& Costa, 2016). O programa "Para mulheres na ciência", fruto de uma parceria público-privada, também contribui para a causa no sentido de aumentar a visibilidade de mulheres cientistas (Quadro 2). Infelizmente, constata-se que iniciativas como essas, voltadas para a mulher que já está inserida no mercado de trabalho, ainda são consideradas escassas no contexto brasileiro. 


\section{Quadro 2}

Exemplos de Programas para Mulheres em STEM

\begin{tabular}{|c|c|c|c|c|}
\hline $\begin{array}{l}\text { Programas/ } \\
\text { Premiações }\end{array}$ & Objetivos & Duração & $\begin{array}{l}\text { Orgão(s) de } \\
\text { Fomento }\end{array}$ & Ações \\
\hline Mulher e Ciência & $\begin{array}{l}\text { Promover a participação das } \\
\text { mulheres nas ciências e } \\
\text { carreiras aca dêmicas, e a } \\
\text { reflexão a cerca das relações } \\
\text { de gênero, mulheres e } \\
\text { feminismos no País. }\end{array}$ & $\begin{array}{l}2005- \\
2017\end{array}$ & $\begin{array}{l}\text { CNPq; } \\
\text { CAPES; } \\
\text { FINEP; } \\
\text { ANDIFES; } \\
\text { UNESCO }\end{array}$ & $\begin{array}{l}\text { Criação do Prêmio Construindo a } \\
\text { Igualda de de Gênero; abertura de } \\
\text { editais para seleção de pesquisas } \\
\text { para apoio financeiro liga do às } \\
\text { temáticas de gênero; criação do } \\
\text { Encontro Pensando Gênero. }\end{array}$ \\
\hline $\begin{array}{l}\text { Para Mulheres na } \\
\text { Ciência }^{6}\end{array}$ & $\begin{array}{l}\text { Incentivar a entrada de } \\
\text { mulheres no universo } \\
\text { científico. Anualmente sete } \\
\text { pesquisa doras de diversas } \\
\text { áreas são contempladas com } \\
\text { uma bolsa-auxilio para sua } \\
\text { pesquisa. }\end{array}$ & $\begin{array}{l}2006- \\
\text { Atualme } \\
\text { nte }\end{array}$ & \begin{tabular}{l|} 
L'oreal \\
Brasil; \\
UNESCO; \\
Academia \\
Brasileira de \\
Ciências.
\end{tabular} & $\begin{array}{l}\text { O prêmio já reconheceu } 82 \\
\text { cientistas promissoras, que } \\
\text { receberam impulso extra para dar } \\
\text { prosseguimento emseus estudos e } \\
\text { incrementar o desenvolvimento da } \\
\text { ciência no Brasil. }\end{array}$ \\
\hline
\end{tabular}

\section{Conclusão}

O presente artigo buscou apresentar e discutir estratégias relevantes para o avanço de mulheres nas carreiras em STEM. Conforme discutido, os principais fenômenos que geram a pouca participação das mulheres em carreiras relacionadas à STEM são o teto de vidro, o efeito tesoura e o leaky pipeline, uma vez que contribuem para a segregação vertical e explicitam a evasão das mulheres ao longo da construção de suas carreiras, sendo tal evasão especialmente crítica no caso das áreas de STEM. Visando enfrentar as estatísticas e contribuir para a identificação de variáveis que permitam o aumento e permanência de mulheres em STEM, são propostas ações desde a educação fundamental, passando pela graduação e atingindo a entrada no mercado e o desenvolvimento da carreira, contando com possibilidades referentes tanto ao contexto quanto a questões internas às mulheres.

A revisão realizada pode contribuir para o desenvolvimento de ações e intervenções, além de programas e workshops que ofereçam às mulheres oportunidades para reconhecer e 
localizar seus pontos fortes, ajudando-as a lidar com as barreiras encontradas no avanço em suas carreiras nas áreas de STEM. Adicionalmente, este artigo contribui para avanços do desenvolvimento de carreira das mulheres em STEM, pois nele observou-se a ausência de programas com esse objetivo no Brasil, sendo que as iniciativas aqui apresentadas podem auxiliar na criação de programas eficazes.

Contudo, o artigo em questão apresenta limitação quanto a estudos e programas nacionais já aplicados, uma vez que estes necessitam de orientações mais estruturadas sobre a maneira que ocorreram, assim como a dificuldade de propor soluções que não envolvam reflexões acerca da sociedade patriarcal, necessárias a qualquer mudança. Dessa forma, pesquisas futuras podem explorar as ações, programas e workshops que já foram desenvolvidos e aplicados, a fim de que se apresentem soluções práticas que contribuam para o avanço das mulheres nas carreiras em STEM e diminuição das estatísticas atualmente desfavoráveis.

${ }^{1}$ Chamada Nº 18/2013 MCTI/CNPq/SPM-PR/Petrobras - Meninas e Jovens Fazendo Ciências Exatas, Engenharias e Computação. Disponível em: http://cnpq.br/chamadaspublicas?p_p_id=resultadosportlet_WAR_resultadoscnpqportl et_INSTANCE_0ZaM\&filtro $=$ abertas $\&$ detalha $=$ chamadaDivulgada\&idDivulgacao $=4341$

2 Programas e ações. SPM. Disponível em: http://www.spm.gov.br/assuntos/educacaocultura-e-ciencia/programas-acoes

3 Chamada CNPq/MCTIC No 31/2018 - Meninas nas Ciências Exatas, Engenharias e Computação. Disponível em: http://www.cnpq.br/web/guest/chamadaspublicas?p_p_id=resultadosportlet_WAR_resultadoscnpqportlet_INSTANCE_0ZaM\&idDiv ulgacao $=8402 \&$ filtro $=$ abertas $\&$ detalha $=$ chamadaDetalhada $\&$ id $=47-1198-5840$

4 Informações retiradas do site "Elas nas Exatas". Disponível em: http://www.fundosocialelas.org/elasnasexatas/inicio

6 Programa Nacional. Disponível em: https://www.paramulheresnaciencia.com.br/opremio/programa-nacional/ 


\section{Referências}

Agrello, D. A., \& Garg, R. (2009). Mulheres na física: poder e preconceito nos países em desenvolvimento. Revista Brasileira de Ensino de Física, 37(1), 1305.1-1305.6. https://doi.org/10.1590/S1806-11172009000100005

Alves, D. M. (2017). A mulher na ciência: desafios e perspectivas. Revista do Programa de Pós Graduação da Unesc: Criar Educação, 7(2), 1-25. http://doi.org/10.18616/ce.v6i2.3232

Altman, Y., Simpson, R., Baruch, Y., \& Burke, R. J. (2005). Reframing the 'glass ceiling' debate. In R. J. Burke \& M. C. Mattis (Eds.), Supporting Women's Career Advancement: Challenges and Opportunities (pp. 58-84). Massachussets: Edward Elgar Publishing, Inc.

Amorin, V. G., Dantas, M., \& Carvalho, M. E. P. (2017). Estratégias de Superação Utilizadas por mulheres recém-doutoras em física. Anais do $13^{\circ}$ Mundos de Mulheres e $11^{\circ}$ Seminário Internacional Fazendo Gênero: Transformações, Conexões, Deslocamentos, Florianópolis, 1-11.

Astin, H. S., \& Sax, L. J. (1996). Developing Scientific Talent in Undergraduate Women. In C. Davis, A. B. Ginorio, C. S. Hollenshead, B. B. Lazarus, P. M. Rayman, \& Associates (Eds.), The Equity Equation: Fostering the Advancement of Women in the Sciences, Mathematics, and Engineering (pp. 96-121). San Francisco: Jossey-Bass.

Barthelemy, R. S., McCormick, M., \& Henderson, C. (2016). Gender discrimination in physics and astronomy: Graduate student experiences of sexism and gender microaggressions. Physical Review Physics Education Research, 12(2), 020119.

Bilimoria, D., Joy, S., \& Liang, X. F. (2008). Breaking Barriers and Creating Inclusiveness: Lessons of Organizational Transformation to Advance Women Faculty in Academic Science and Engineering. Human Resource Management, 47, 423-441. https://doi.org/10.1002/hrm.20225

Blickenstaff, J. C. (2005). Women and science careers: leaky pipeline or gender filter?. Gender and education, $17(4)$, 369-386. https://doi.org/10.1080/09540250500145072 
Brainard, S. G., \& Carlin, L. (1998). A Six-Year Longitudinal Study of Undergraduate Women in Engineering and Science. Journal of Engineering Education, 87(4), 17-27. https://doi.org/10.1002/j.2168-9830.1998.tb00367.x

Brito, C., Pavani, D., \& Lima Jr., P. (2015). Meninas na ciência: atraindo jovens mulheres para carreiras de ciência e tecnologia. Revista Gênero, 16(1), 33-50. https://www.if.ufrgs.br/cbrito/publicacoes/RevistaGenero_Brito2015.pdf

Busch-Vishniac, I. J. (2015). Climbing the Ladder. In Pritchard, P. A. \& Grant, C. S. (Eds.), Success Strategies From Women in STEM: A Portable Mentor (2a ed., pp. 365-389). Cambridge: Academic Press.

Cadaret, M. C., Hartungb, P. J., Subich, L. M., \& Weugold, I. K. (2017). Stereotype threat as a barrier to women entering engineering careers. Journal of Vocational Behavior, 99, 4051. https://doi.org/10.1016/j.jvb.2016.12.002

Caseira, F. F., \& Magalhães, J. C. (2017). Meninas em carreiras de ciência e tecnologia: investigando alguns programas brasileiros. Anais do $17^{\circ}$ Seminário Internacional Fazendo Gênero e 13th Women's Worlds Congress, Florianópolis, 01-12.

Caseira, F. F., \& Magalhães, J. C. (2015). "Para mulheres na ciência": uma análise do programa da L'Oréal. Revista Ibero-Americana de Estudos em Educação, 10, $n$. especial, 1523-1544. https://doi.org/10.21723/riaee.v10i6.8335

Catalyst. (2007). The Double-Blind Dilemma for Women in Leadership: Damned If You Do, Doomed If You Don't. New York: Editora Catalyst.

Cheryan, S., Siy, J. O., Vichayapai, M., Drury, B. J., \& Kim, S. (2011). Do Female and Male Role Models Who Embody STEM Stereotypes Hinder Women's Anticipated Success in STEM? Social Psychological and Personality Science, 2(6), 656-664. https://doi.org/10.1177\%2F1948550611405218

Chronister, K., McWhirter, E. H., \& Forrest, L. (2008). A Critical Feminist Approach to Career Counseling With Women. In W. B. Walsh \& M. Heppner (Eds.), Handbook of carrer couseling for women (2a ed., pp. 167-192). New Jersey: Lawrence Erlbawn Associates, Inc.

Clerc, I., \& Kels, P. (2013). Coping with Career Boundaries in Masculine Professions: Career Politics of Female Professionals in the ICT and Energy Supplier Industries in 
Switzerland. Gender, Work and Organization, 20(2), 197-210. https://doi.org/10.1111/gwao.12017

Conselho Nacional de Desenvolvimento Cientifico e Tecnológico (CNPq). 2016. As listas de bolseiros são fornecidas pelo CNPq. Disponível em http:// www.cnpq.br/ web/ guest/bolsistasvigentes. Acesso em 13/10/2021.

Damasceno, A. G. S., \& Bentes, J. L. (2014). O ensino de engenharia para meninas do ensino público como forma de incentivo destas para o curso superior. Anais Engenharia: Múltiplos Saberes e Atuações, Juiz de Fora, 1-11. http://www.abenge.org.br/cobenge/arquivos/5/Artigos/129266.pdf

Ehrlinger, J., \& Dunning, D. (2003). How chronic self-views influence (and potentially mislead) estimates of performance. Journal of Personality and Social Psychology, 84(1), 5-17. https://pubmed.ncbi.nlm.nih.gov/12518967/

Elsevier. (2017). Gender in the Global Research Landscape. Elsevier's Research Intelligence portfolio of products and services serves research institutions, government agencies, funders, and companies. Amsterdã: Elsevier.

Falk, N. A., Rottinghaus, P. J., Casanova, T. N., Borgen, F. H., \& Betz, N. E. (2017). Expanding Women's Participation in STEM: Insights From Parallel Measures of Self-Efficacy and Interests. Journal of Career Assessment, 25(4), 571-5840. https://doi.org/10.1177\%2F1069072716665822

Fouad, N. A., Singh, R., Cappaert, K., Chang, W., \& Wan, M. (2016). Comparison of women engineers who persist in or depart from engineering. Journal of Vocational Behavior, 92, 79-93. https://doi.org/10.1016/j.jvb.2015.11.002

Grant, C. S. (2015). Mentoring: Empowering Your Success. In P. A. Pritchard, \& C. S. Grant (Eds.), Success Strategies From Women in STEM: A Portable Mentor (2a ed., pp. 63-96). Cambridge: Academic Press.

Greenhaus, J. H., Callanan, G. A., \& Godshalk, V. M. (2010). Career management (4th ed.). Thousand Oaks: SAGE Publications, Inc.

Hawks, B. K., \& Spade, J. Z. (1998). Women and Men Engineering Students: Anticipation of Family and Work Roles. Journal of Engineering Education, 87(3), 249-256. https://doi.org/10.1002/j.2168-9830.1998.tb00351.x 
Herman, C. (2005). Rebooting and Rerouting: Women's Articulations of Frayed Careers in Science, Engineering and Technology Professions. Gender, Work and Organization, 22(4), 324-338. https://doi.org/10.1111/gwao.12088

Hill, C., \& Silva, E. (2005). Drawing the line: sexual harassment on campus. Washington: AAUW Educational Foudation. http://files.eric.ed.gov/fulltext/ED489850.pdf

Huang, P. M., \& Brainard, S. G. (2001). Identifying Determinants of Academic Self-Confidence Among Science, Math, Engineering, and Technology Students. Journal of Women and Minorities in Science and Engineering, $7(4), \quad 315-337$. https://doi.org/10.1615/JWomenMinorScienEng.v7.i4.40

Kinzie, J. (2007). Women's Paths in Science: A Critical Feminist Analysis. New Directions for Institutional Research, 133, 81-93. https://doi.org/10.1002/ir.206

Klanovicz, L. R. F. (2011). Gênero e engenharias: um estudo histórico quali-quantitativo da inserção, permanência e produção científica de mulheres no sul do Brasil [Relatório final pós-doutorado DICH, Universidade Federal de Santa Catarina].

Klanovicz, L. R. F. (2016). A visibilidade de mulheres cientistas na imprensa brasileira: gênero, história da ciência e ressonâncias do feminismo. Anais do $16^{\circ}$ Encontro Estadual de História da Anpuh, Santa Catarina, 1-11. http://www.encontro2016.sc.anpuh.org/resources/anais/43/1464644292_ARQUIVO_ KLANOVICZlucianaANPUHSC.pdf

Lima, B. S., Braga, M. L. S., \& Tavares, I. (2015). Participação das Mulheres na Ciência e Tecnologia: entre espaços ocupados e lacunas. Revista Gênero, 16(1), 11-31. https://doi.org/10.22409/rg.v16i1.743

Lima, B. S., \& Costa, M. C. (2016). Gênero, ciências e tecnologias: caminhos percorridos e novos desafios. Cadernos Pagu, 48, 1-39. http://doi.org/10.1590/18094449201600480005

Lima, G. S., Carvalho Neto, A., Lima, M. S., Tanure, B., \& Versiani, F. (2013). O teto de vidro das executivas brasileiras. Pretexto, 14(4), 65-80. https://doi.org/10.21714/pretexto.v14i4.1922 
Lombardi, M. R. (2003). Mulheres engenheiras no mercado de trabalho brasileiro: qual seu $\begin{array}{lllll}\text { lugar? } & \text { Mulher } & \text { e } & \text { Trabalho, } & 45-59 \text {. }\end{array}$ http://revistas.planejamento.rs.gov.br/index.php/mulheretrabalho/article/view/2700 Lyon, G. H., Jafri, J., \& Louis, K. St. (2012). Beyond the pipeline: STEM Pathways for Youth Development. Afterschool Matters, (16), 48-57. https://eric.ed.gov/?id=EJ992152

Luyhans, F., Yousseff, C. M., \& Avolio, B. J. (2007). Psychological capital. Oxford: Oxford University Press.

Margolis, J., Fisher, A., \& Miller, F. (2000). The Anatomy of Interest: Women in Undergraduate Computer Science. Women's Studies Quarterly, 28(1/2), 104-127. https://www.jstor.org/stable/40004448

McCauley, P., \& Lee, E. (2015). A call to Leadership. In P. A. Pritchard \& C. S. Grant (Eds.), Success Strategies From Women in STEM: A Portable Mentor (2a ed., pp. 378-407). Cambridge: Academic Press.

Morrison, A. M., \& Von Glinow, M. (1990). Women and minorities in management. American Psychologist, 45, 200-208.

Muzi, J. L. C., \& Luz, N. S. (2010). Entre cursos e discursos: um olhar sobre mulheres e Ciência na Universidade. Anais do $8^{\circ}$ Congresso Iberoamericano de Ciência, Tecnologia e Gênero, Curitiba, 1-12.

National Steering Committee for Career Development Guidelines and Standards. (2012). Glossary of career development terms. Canadian Standards and Guidelines for Career Development Practitioners. http://career-devguidelines.org/career_dev/index.php/the-standards-guidelines/glossary-of-terms

Nosek, B. A., Banaji, M. R., \& Grennwald, A. G. (2002). Math Male, Me Female, Therefore Math Me. Journal of Personality and Social Psychology, 83(1), 44-59. https://doi.org/10.1037/0022-3514.83.1.44

Olinto, G. (2011). A inclusão das mulheres nas carreiras de ciência e tecnologia no Brasil. Inclusão Social, 5(1), 68-77. http://revista.ibict.br/inclusao/article/view/1667

Paiva, A. P. (2017). Divisão sexual do Trabalho e Teto de Vidro: o desenvolvimento da carreias de mulheres cientistas. Anais do $11^{\circ}$ Seminário Internacional Fazendo Gênero \& $13^{\circ}$ Women's Worlds Congress, Florianópolis, 1-12. 
Pritchard, P. A. (2015). Career Management. In P. A. Pritchard \& C. S. Grant (Eds.), Success Strategies From Women in STEM: A Portable Mentor (2a ed., pp. 46-57). Cambridge: Academic Press.

Reuben, E., Rey-Biel, P., Sapienza, P., \& Zingales, L. (2012). The emergence of male leadership in competitive environments. Journal of Economic Behavior \& Organization, 83(1), $111-117$. https://doi.org/10.1016/j.jebo.2011.06.016

Rosa, K., \& Silva, M. R. G. (2015). Feminismos e ensino de ciências: análise de imagens de livros didáticos de física. Revista Gênero, 16(1), 83-104. https://doi.org/10.22409/rg.v16i1.747

Rummer, J. L. (2015). Networking. In P. A. Pritchard \& C. S. Grant (Eds.), Success Strategies From Women in STEM: A Portable Mentor (2a ed., pp. 68-107). Cambridge: Academic Press.

Saavedra, L., Taveira, M. C., \& Silva, A. D. (2010). A subrepresentatividade das mulheres em áreas tipicamente masculinas: factores explicativos e pistas para a intervenção. Revista Brasileira de Orientação Profissional, $\quad l l(1), \quad$ 49-59. http://pepsic.bvsalud.org/pdf/rbop/v1 ln1/v1 ln1a06.pdf

Sandler, B. R. (2005). The Chilly Climate. The Big Ten Academic Alliance, 1-7.

Santos, V. M. (2016). Uma "perspectiva parcial" sobre ser mulher, cientista e nordestina no Brasil. Revista Estudos Feministas, 24(3), 801-824. https://doi.org/10.1590/18069584-2016v24n3p801

Schweitzer, L., Ng, E., Lyons, S., \& Kuron, L. (2011). Exploring the Career Pipeline: Gender Differences in Pre-Career Expectations. Industrial Relations, 66(3), 422-444. https://www.jstor.org/stable/23078364

Seymour, E., \& Hewitt, N. (1997). Talking About Leaving: Why Undergraduates Leave the Sciences. Boulder: Westview Press.

Shapiro, C. A., \& Sax, L. J. (2011). Major Selection and Persistence for Women in STEM. Wiley Periodicals, 152, 5-18. https://doi.org/10.1002/ir.404

Vanbrabrant, D. (2012). How do Women Network Differently From Men? Gender Info, 10, 2024. 
Velho, L., \& León, E. (1998). A construção social da produção científica por mulheres. Cadernos Pagu, (10), 309-344. https://periodicos.sbu.unicamp.br/ojs/index.php/cadpagu/article/view/4631474 Vongalis-Macrow, A. (2016, 28 de Setembro). What It Will Take to Keep Women from Leaving STEM? Harvard Business Review. https://hbr.org/2016/09/what-it-will-take-to-keepwomen-from-leaving-stem

Recebido em: 28/9/2018

Aprovado em: $24 / 4 / 2019$ 